\title{
Photo-Elicitation as an Adjunct to Structured Interviews When Assessing Ideal Romantic and Sexual Relationships
}

\author{
Candace Best, J. Dennis Fortenberry, Sarah E. Wiehe
}

\section{Purpose}

Structured interviews have been used as an assessment tool in clinical and research settings for many years. However, such interviews have limitations, especially when questions are abstract from the daily life experiences of adolescents and young adults (e.g., "What are all the qualities you desire in a romantic partner?"). Accordingly, photo-elicitation was incorporated as a tool into how young women perceive ideal romantic and sexual relationships.

\section{Methods}

Participants included 13 African American heterosexual young women (ages 14-17) recruited from the local community in a mid-sized Midwestern city. Young women met with the interviewer once at a private location for approximately 1.5 hours. During the interview, young women were asked to consider their ideal partner by thinking of "someone you could be with in an ideal world." They were asked to think carefully about what that person might look like, etc. Participants were provided with a 24-item list of qualities representative of an ideal partner. The list utilized ideal traits described in previous research studies (e.g., "cute face," "supportive," "certain skin-complexion"). Participants were then handed photographs ( $N=$ up to 98 ) in random order. Photographs were obtained from publicallyavailable online resources and were purposefully chosen to highlight a range of ethnicities, behaviors and personality attributes of young men (e.g., "African American man smiling," "interracial couple shopping"). Young women were then asked to identify which ideal partner qualities they observed in each picture. Young women were permitted to state as many or as few items as they desired and they could state any additional partner quality not included on the list. Vague or extensive responses were probed when applicable. At the end of the study, participants provided feedback of their experiences. Interviews were recorded, transcribed and coded using grounded theory.

\section{Results}

When evaluating all responses, 5 themes emerged relating to this technique. First, young women were able to quickly identify partner qualities when provided with a photograph. Second, participants rapidly described ideal partner qualities that were not included on the 24-item list. Third, young women articulated elaborate stories about the young men depicted in the pictures, often with minimal to no probing. Fourth, participants indicated that they enjoyed the activity and that it was "easy" to provide the requested information. Fifth, most were able to identify at least one picture that was representative of their ideal partner. Three unexpected and unsolicited findings from this project are noteworthy. Specifically, young women almost unanimously indicated that participating in this study helped them

This is the author's manuscript of the article published in final edited form as:

Best, C., Fortenberry, J. D., \& Wiehe, S. E. (2013). 103. Photo-Elicitation as An Adjunct to Structured Interviews When Assessing Ideal Romantic and Sexual Relationships. Journal of Adolescent Health, 52(2, Supplement 1), S71. Available from: http://dx.doi.org/10.1016/j.jadohealth.2012.10.165 
clarify the qualities they desire in an ideal partner. Second, a number of participants revealed elaborate criteria when evaluating the skin complexion of an ideal partner. Finally, participants spoke honestly about their desire for marriage in their future.

\section{Conclusions}

Adolescent women were very comfortable conversing openly about ideal romantic and sexual relationships when using photographs as tools. Without incorporation of the photographs, it is questionable how much unsolicited information would have been garnered. Overall, photo-elicitation is a complimentary adjunct to structured interviews with both research and clinical implications.

\section{Sources of Support}

T-32 Postdoctoral Training Grant. 